doi:10.1017/S0022215116002139

\section{Middle ear inflammation, mucin gene expression and hearing loss: bench to bedside (K655)}

\section{ID: 655.1}

Middle ear inflammation, mucin gene expression and hearing loss: bench to bedside

Presenting Author: Joseph Kerschner

Joseph Kerschner

Medical College of Wisconsin

Otitis media $(\mathrm{OM})$ remains one of the most important and common diseases of childhood. With decreased effectiveness of antimicrobial therapy our laboratory has spent the past 2 decades elucidating the mechanisms surrounding the pathophysiology of OM in hopes of identifying novel methods of intervention. Through this work, substantive advances in the understanding of the pathogenesis, management and treatment of OM continue to develop. This presentation will highlight the most substantive of these developments in the areas of middle ear inflammation, mucin gene expression, biofilm formation and hearing loss associated with OM. It will provide the clinician and researcher with a platform to: 1) facilitate discussion of new concepts with patients and colleagues, 2) allow for incorporation of new modalities into their practice and 3) remain up-to-date regarding clinical and research developments that have the potential to provide new technologies and treatment possibilities.

doi:10.1017/S0022215116002140

\section{Emerging Technologies (2) (R661)}

\section{ID: 661.1}

\section{Stereotactic robotic system for ear surgery}

\section{Presenting Author: Thomas Lenarz}

Thomas Lenarz ${ }^{1}$, Omid Majdani ${ }^{1}$, Samuel John ${ }^{2}$

${ }^{1}$ Medical University Hannover, ${ }^{2} \mathrm{HörSys} \mathrm{GmbH}$

Learning Objectives: Robot assited cochlear implantation.

Background: Cochlear implantation has become the treatment of choice for patients with severe to profound sensory hearing loss. An increasing percentage of patients show up with residual hearing which should be preserved during and after surgery. In order to improve the precision of electrode insertion with respect to individual cochlear anatomy and existing hearing loss, an advanced concept of robot assisted cochlear implantation has been developed.

Method: The stereotactic frame robotic system has been developed to allow for minimal invasive high precision cochlear implantation. Using preoperative CT data and a tripod stereotactic frame it is possible to calculate precisely the optimal trajectory from the surface of the mastoid to the inner ear. This path can then be drilled with help of a disposable drilling jig that, which has been individualized to the planned trajectory with an intraoperative robotic assistance system and it has been validated in temporal bone and surgeon studies prior to planned first in man applications.

Results: The overall accuracy is better than 0.5 millimeter. Injuries to facial nerve and scala tympani can be avoided with high probability. The exact placement of the electrode in the scala tympani was achieved.

Conclusion: Robot assisted ear surgery, especially cochlear implantation is both feasible and possible. Introducing such a system would decrease the time needed for implantation by half. The system is easy to use with minimal requirements in terms of technology. It can be adapted for a wide range of auditory implants.

doi:10.1017/S0022215116002152

\section{Emerging Technologies (2) (R661)}

\section{ID: 661.2}

\section{Microsurgical robot for direct cochlea access}

\section{Presenting Author: Marco Caversaccio}

Caversaccio $\mathrm{M}^{1,2}$, Williamson $\mathrm{T}^{3}$, Gavaghan $\mathrm{K}^{2}$, Gerber $\mathrm{N}^{2}$, Wimmer $\mathrm{W}^{1,2}$, Anso $\mathrm{J}^{3}$, Rathgeb $\mathrm{C}^{2}$, Wagner $\mathrm{F}$, Weber $\mathrm{S}^{2}$

${ }^{1}$ Department of ENT, Head and Neck Surgery, University Hospital, Bern, Switzerland ${ }^{2}$ ARTORG Center, Bern, Switzerland

\section{Learning Objectives:}

Objectives: Over the last decade significant research efforts have been invested towards the application of surgical robotics for cochlear implant surgery. To this end, a high accuracy image guided robotic manipulator was developed at the University of Bern, allowing minimally invasive access to the cochlea. This work aims to evaluate the accuracy and usability of the developed system in a study on human temporal specimens.

Methods: A complete surgical workflow for robotic assisted minimally invasive cochlear implantation, including fiducial screw insertion, pre-operative imaging, semi-automatic trajectory planning, patient-to-image registration, tunnel drilling, and electrode array insertion was developed. The proposed workflow, the accuracy of the robotic system, and the effectiveness of the integrated safety features were evaluated in a total of 22 human temporal bone specimens.

Results: In all cases, access to the cochlea was successfully obtained. A drilling accuracy of $0.15 \pm 0.07 \mathrm{~mm}$ was observed at the round window of cochlea as determined from post-operative image data. The additional mechanisms provide a means to monitor the safety of the approach during the surgery. The system is approved by the Swissmedic regulatory and the Swiss Ethical Committee has given permission for a first in man clinical trial. 
Conclusions: The robotic system allows the accurate and safe drilling of a minimally invasive tunnel to the inner ear for cochlear implantation procedures. The evaluation of the system in a first in man clinical trial will take place in the near future.

Acknowledgments: Artorgcenter (Prof. S. Weber and coworkers), Medel-company, Nano-Tera, CTI, Hear-EU.

doi:10.1017/S0022215116002164

\section{Emerging Technologies (2) (R661)}

\section{ID: 661.3}

The Case for Cochlear implantation

Robotics and an autonomous drilling robot

Presenting Author: Chris Coulson

Chris Coulson

Queen Elizabeth Hospital, Birmingham. endoscope- $i$

Learning Objectives: Cochlear implantation leads to cochlear trauma, reducing this may help optimize implantation performance. An autonomous cochlea drilling robot may be one of the components in reducing this trauma.

Introduction: To detail the intra cochlear trauma caused during cochlear implantation and its effect on CI performance. To demonstrate a human trial of an autonomous robot capable of performing a bony cochleostomy whilst preserving the underlying endosteal membrane.

Methods: A review of the implantation literature assessing cochlear trauma and its impact on implant performance. An autonomous cochleostomy robot was used to create a cochleostomy in 3 live patients during a cochlear implantation procedure.

Results: Twenty one papers were identified which were relevant to our search. In total, 686 implants were inserted and $121(17.6 \%)$ showed evidence of trauma.

The robotic cochleostomy drilling robot was able to perform a complete cochleostomy whilst preserving the underlying endosteal membrane.

Conclusions: Cochlea trauma is a common result of cochlear implantation. An autonomous robotic drill can perform a cochleostomy whilst preserving the underlying endosteal membrane. This is one of the necessary steps in being able to perform a completely robotic cochlear implantation with an intention to reduce the typical cochlear trauma.

doi:10.1017/S0022215116002176

Free Papers (F662)

ID: 662.1

Middle fossa approach for cochlear implantation
Presenting Author: Wojciech Gawęcki

Wojciech Gawęcki ${ }^{1}$, Łukasz Borucki ${ }^{1}$, Michał Karlik ${ }^{1}$, Vittorio Colletti ${ }^{2}$, Witold Szyfter ${ }^{1}$

${ }^{1}$ Poznań University of Medical Sciences,

${ }^{2}$ University of Verona

Learning Objectives: To present indications, surgery and results of cochlear implantation via middle fossa approach.

Introduction: Classic approach to the cochlea through the mastoid and facial recess may not be suitable for patients after middle ear surgery for cholesteatoma. In 1998 Colletti presented a technique to bypass the middle ear, and insert the electrode through the middle cranial fossa approach.

Material and methods: In our department cochlear implant program started in 1994 and till now 1266 cochlear implantations were performed. In 4 patients middle fossa approach was used. Indications, surgical technique and results in this group were analyzed. Results: Initially 6 deaf patients after middle ear operation for cholesteatoma were qualified to cochlear implantation via middle fossa approach. A middle cranial fossa craniectomy was performed. Then a careful dissection of the dura was carried out to expose the arcuate eminence and the greater petrosal nerve. In two cases surgery was stopped because of strong adhesions and bleeding from the dura during preparation. In the rest 4 cases the basal turn of the cochlea was discovered, the cochleostomy was done and successful implantation was performed. The time of surgery was nearly two times longer than during standard implantation. In 3 cases there were no complications and in one case hematoma occurred 2 days after surgery and the patient was reoperated. Postoperative CT showed correct intracochlear position of the electrode in all cases. All 4 patients use their implants and have good hearing thresholds in sound free field, but they can't fully communicate using hearing only and require lip reading.

Conclusions: Middle fossa approach enables cochlear implantation in deaf patients after middle ear surgery where implantation through standard approach (antromastoidectomy and posterior tympanotomy) is not possible.

doi: $10.1017 / \mathrm{S} 0022215116002188$

Free Papers (F662)

\section{ID: 662.2}

Systematic Review of VSB in C/M Hearing Loss

Presenting Author: Arne Ernst

Arne Ernst ${ }^{1}$, Jan Wagner ${ }^{2}$

${ }^{1}$ Charité Medical School, ${ }^{2}$ dept otolaryngology at ukb, hospital of the univ. of berlin (Charité)

Pubmed, OvidSP (MEDLINE), EMBASE (DIMDI), the NHR Centre for Reviews and Dissemination (including NHS EED, DARE, and HTA), and the Cochrane Library were searched to identify papers published between January 2006 and December 2015 using the MeSH terms 